\title{
TRASTORNOS ENDOCRINOS DURANTE LA PUBERTAD
}

\author{
Dr. Arturo Zárate* \\ Dr. Carlos MacGregor** \\ Dr. Luis Castelazo-Ayala** \\ Dr. Elias S. Canales***
}

Hospital de Gineco-Obstetricia No 1 - Instituto Mexicano del Seguro Social, México.

Los trastornos que se presentan en la mujer durante la adolescencia y la pubertad siempre despiertan un gran interés y con mucha frecuencia se interpretan inadecuadamente. Es una época en la cual la paciente sale del dominio del pediatra y aún no ha alcanzado la edad para ser estudiada por el internista. Debido a que los trastornos involucran fundamentalmente el desarrollo sexual y la menstruación, es el ginecólogo quien con mayor frecuencia estudia y trata los problemas de la adolescencia.

Se entiende por pubertad el período que comprende desde el inicio de la maduración sexual hasta la primera menstruación o menarca, época en la que clínicamente se completa la diferenciación sexual. En este período se realizan numerosos cambios y ajustes endocrinos cuyo mecanismo íntimo aún tiene muchos puntos oscuros, que han sido motivo de gran atención en los últimos años.

\section{Diferenciación sexual.}

En términos muy generales la diferenciación sexual es el resultado de 4 estadios sucesivos.
1. Diferenciación gonadal. Aparentemente se realiza por la influencia del componente cromosómico del individuo. En el caso de tratarse de un sujeto con un cariotipo 46, XY Y por lo tanto destinado a ser un hombre, la gónada indiferenciada que se encuentra en la raíz dorsal del mesenterio sufre cambios alrededor de la semana cuarta de la vida fetal, consistentes en atrofia de su porción cortical y proliferación de la medular, formándose así un testículo. En cambio, cuando el componente cromosómico es de $46 \mathrm{XX}$, los cambios en la gónada primitiva consisten en la atrofia de la porción medular y un desarrolo de la cortical lo cual origina un ovario (1).

2. Diferenciación de los genitales internos. El feto posee 2 tipos de estructuras, los conductos de Müller y los de Wolff los cuales darán origen a los genitales internos de uno $u$ otro sexo, dependiendo de la presencia o no de testículo. Cuando hay testícu-

\footnotetext{
* Profesor de Endocrinología.

*** Profesor de Ginecología y Obstetricia.

*** Profesor Asistente de Ginecología y Obstetricia. Universidad Nacional Autónoma de Mé-
} xico. 
lo, se secreta un factor, de naturaleza aún desconocida, el cual produce desaparición de los conductos de Müller y desarrollo de los de Wolff dando así origen a genitales internos del sexo masculino. En ausencia de testículo, haya o no ovario, las estructuras de Müller dan origen a las trompas de Falopio, al útero y al tercio superior de la vagina, atrofiándose simultáneamente los conductos de Wolff (1).

3. Diferenciación de los genitales externos. Esta etapa depende directamente del efecto de los andrógenos, posiblemente testosterona, sobre los genitales externos primitivos haciendo que adquieran características masculinas. En ausencia de una acción androgénica, independientemente de la composición cromosómica y de la naturaleza de la gónada, el desarrollo de los genitales externos es de tipo femenino (1).

\section{La pubertad es la culminación} de la diferenciación sexual. En esta época se instala activamente la unidad hipotálamo-hipófisis-ovario.

\section{Fisiología de la Pubertad.}

Aunque se han propuesto innumerables mecanismos y explicaciones para la instalación de la pubertad en todos se encuentran dudas y hasta la actualidad permanece oscuro este fenómeno biológico. Las consideraciones más importantes que se pueden hacer son las siguientes:

Desde antes de la pubertad parece existir una interrelación activa de la unidad hipotálamo - hipófisis - ovario; esto basado tanto en observaciones recogidas en experimentos en animales como estudios en el humano (2). Además en los animales, se ha encontrado que el ovario pasa por 2 fases: una temprana durante la cual el ovario parece ser insensible a las gonadotropinas, y otra posterior, en la que la gónada responde al estímulo.

En el humano durante la etapa prepúber se ha demostrado, tanto en orina como en sangre, que existen gonadotropinas circulantes (3). Existe mayor concentración de hormona estimulante del folículo (FSH) que hormona luteinizante ( $\mathrm{LH}$ ) y aunque estas hormonas van aumentando con la edad, muestran pocas fluctuaciones diarias. Por otro lado, las observaciones en animales no se han confirmado en el humano, de aquí que lo único que se ha llegado a concluir es que el inicio de la pubertad se manifiesta como un aumento en la secreción de gonadotropinas, especialmente $\mathrm{LH}$, con punto de partida en el hipotálamo pero sin saberse cual es el "iniciador" y sólo se especula si este proviene de centros nerviosos superiores o bien de los ovarios.

La primera menstruación o menarca es la manifestación clínica evidente de una interrelación hipotálamohipófisis-ovario, completamente desarrollada. Al principio las menstruaciones son irregulares y así permanecen hasta que se instala la ovulación, lo cual confiere una "ciclicidad" menstrual.

\section{Trastornos endocrinos en la Adolescencia.}

Los principales trastornos que se observan en la adolescencia son:

I. Falla en la presentación de la pubertad.

11. Pubertad precoz. tad. 
IV. Amenorrea primaria con desarrollo sexual.

\section{Ausencia de Pubertad.}

Una falla para presentarse la última etapa del desarrollo sexual puede obedecer fundamentalmente a 3 causas:

1. Trastorno en la secreción de gonadotropinas.

2. Alteración gonadal.

3. Insensibilidad de los tejidos efectores a las hormonas.

4. Amenorrea primaria y ovarios poliquísticos.

1. Anormalidad en la secreción de gonadotropinas. Esto se puede obser- var en casos de un tumor hipofisiario en el que hay una destrucción de las células productoras de FSH y LH, que puede ser la primera manifestación de la neoplasia adenohipofisiaria (4). También se han descrito lesiones tumorales de asiento hipotalámico asociadas a un infantilismo sexual. El craniofaringioma sigue siendo el tumor que más frecuentemente ocasiona hipogonadismo en las niñas.

Existe otro cuadro patológico que, sin demostrarse una lesión orgánica, cursa con ausencia selectiva de gonadotropinas y frecuentemente tiene un carácter familiar (Tabla 1). Cuando afecta a personas del sexo masculino suele asociarse con anosmía y criptorquidia; en la mujer, los tras-

TABLA 1

PRINCIPALES CARACTERISTICAS CLINICAS EN TRES HERMANOS CON DEFICIENCIA SELECTIVA DE GONADOTROPINAS HIPOFISIARIAS (HIPOGONADISMO HIPOGONADOTROPICO FAMILIAR)

\begin{tabular}{lccccccc}
\hline Caso & Sexo & Edad & Talla & Infantilismo & Anosmia & Hipotiroidismo & Exploración \\
\hline JM & F & 20 & 1.55 & si & no & ausente & $\mathrm{N}$ \\
RM & M & 17 & 1.68 & si & sí & ausente & $\mathrm{N}$ \\
DM & M & 16 & 1.50 & sí & no & ausente & $\mathrm{N}$ \\
\hline
\end{tabular}

tornos del olfato son excepcionales (5). Durante mucho tiempo se ignoró la naturaleza del trastorno y solo recientemente se ha encontrado que la administración de hormonas hipotalámicas liberadoras de LH es capaz de producir un estímulo sobre la adenohipófisis con la consecuente secreción de FSH y LH (6) (Tabla 2 ). El tratamiento de este problema se ha tornado muy simple en la actualidad puesto que la feminización se puede lograr adecuadamente con el uso de estrógenos y la ovulación con fines de embarazo se consigue tanto con hormonas hipotalámicas como con gonadotropinas exógenas.
TABLA 2

DETERMINACION DE HORMONA LUTEINIZANTE EN EL PLASMA DESPUES DE LA ADMINISTRACION DE LH-RH* EN TRES PACIENTES CON DEFICIENCIA SELECTIVA DE GONADOTROPINAS

\begin{tabular}{cccc}
\hline $\begin{array}{c}\text { TIEMPO } \\
\text { ( } \mathbf{m i n})\end{array}$ & J M & $\begin{array}{c}\text { L H } \\
(\mathbf{m U l} / \mathbf{m l}) \\
\mathbf{R ~} \mathbf{M}\end{array}$ & D M \\
\hline 0 & 3.6 & 1.2 & 1.2 \\
8 & 14.4 & 3.0 & 2.9 \\
16 & 27.0 & 2.6 & 1.9 \\
32 & 30.0 & 2.5 & 2.2 \\
64 & 16.8 & 1.8 & 1.3 \\
128 & 10.7 & 1.2 & 4.1 \\
192 & 6.3 & 1.4 & 4.1 \\
* LH-RH 300 ug IV. & & \\
\hline
\end{tabular}


2. Alteración Gonadal. Cualquier trastorno que impida el desarrollo gonadal provoca un hipogonadismo que frecuentemente se descubre hasta la adolescencia. Las variedades más frecuentes son las siguientes:

a) Disgenesia gonadal con fenotipo de Turner. Aparentemente la falta de un cromosoma sexual hace que no se formen las gónadas y por tal motivo se desarrollan subsecuentemen- te las estructuras de Müller y los genitales externos son de tipo femenino. La alteración cromosómica también acarrea malformaciones somáticas congénitas que permiten hacer el diagnóstico junto con el análisis citogenético, prácticamente desde el nacimiento. El tratamiento se restringe a la administración de una terapia de substitución con estrógenos y la advertencia de la imposibilidad de fertilidad.

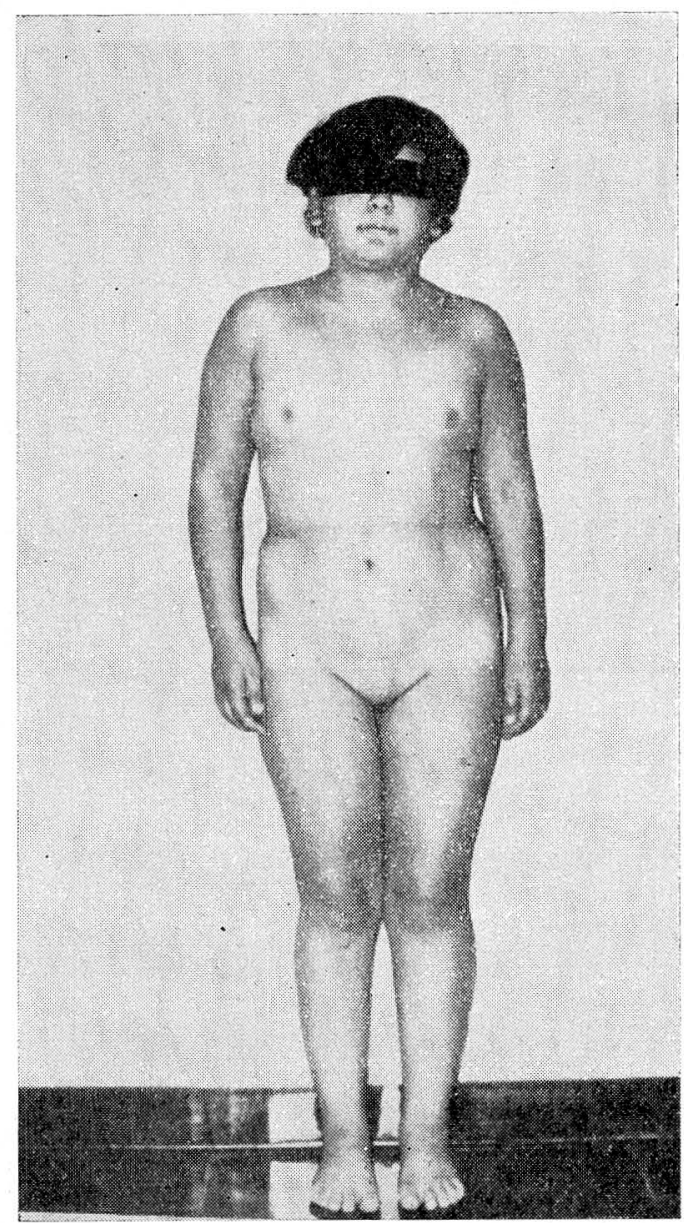

FIGURA 1 - Paciente de 18 años de edad con disgenesia gonadal pura en la que no existe desarrollo sexual ni anomalías somáticas. 
b) Disgenesia gonadal pura. En ocasiones no se llega a formar la gónada a pesar de tener una composición cromosómica completa. En tales casos el desarrollo del individuo, independientemente del cariotipo que posea sigue la línea femenina y debido a la ausencia de malformaciones congénitas, el diagnóstico no se realiza hasta que la paciente asiste a consulta por la falta de pubertad (Fig. 1). La demostración de una ausencia de gónadas mediante estudios radiológicos (Fig. 2) o endoscópicos, confirma el diagnóstico y de inmediato se instituye medicación hormonal sexual substitutiva para feminizar y provocar sangrados menstruales, lo cual psicológicamente tiene gran ayuda para la paciente (7).

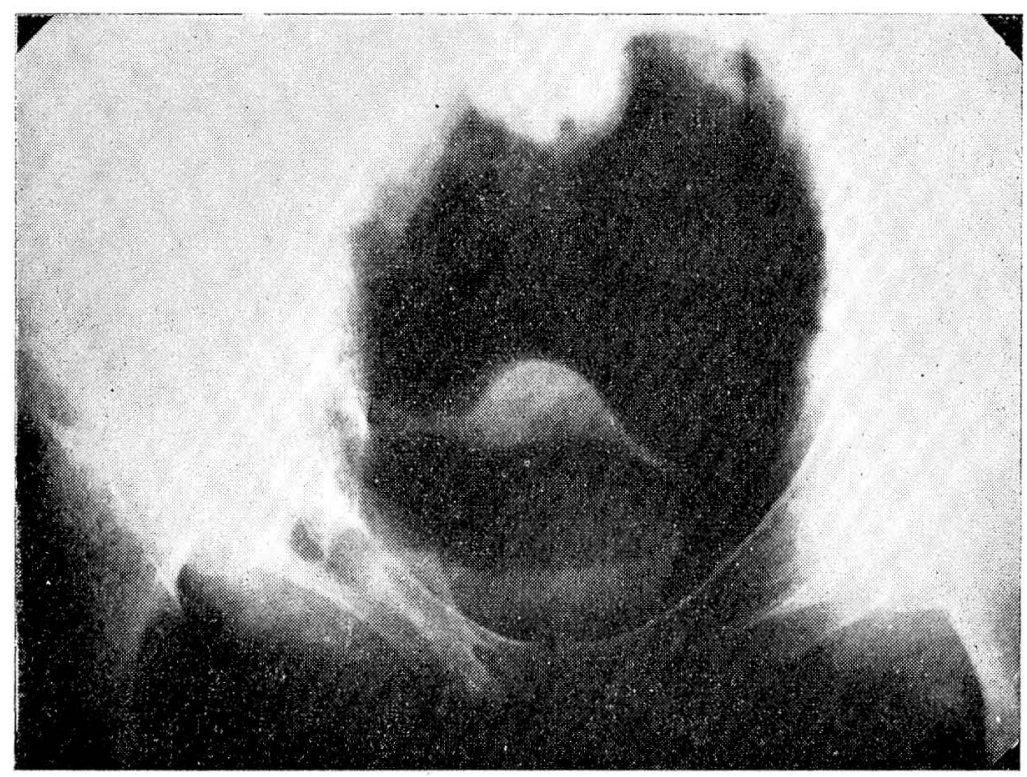

FIGURA 2 - Nótese en esta pelvineumografía la presencia de útero y trompas pero sin ovarios.

c) Disgenesia gonadal mixta. Esta entidad representa un trastorno muy importante en la diferenciación gonadal que puede tener muchas variantes ya que puede tratarse de: 1) ausencia gonadal en un lado y testículo $u$ ovario en el otro lado, y 2) neoplasia gonadal de un lado y gónada diferenciada del lado contralateral. Las características de los genitales internos dependerán de la variedad que se trata y los genitales características de ambos sexos. Las características clínicas relevantes, que inclusive permiten hacer el diagnóstico, con un individuo con fenotipo femenino, infantilismo sexual y clitoromegalia (Fig. 3). La demostración de una cromatina sexual negativa o un cariotipo 46, XY confirma el diagnóstico. En estos casos la existencia de neoplasias malignas de la gónada disgenética hace imperativo practicar una laparotomía para extirpar ya sea el vestigio de gónadas o bien el tumor existente (8). Tam- 


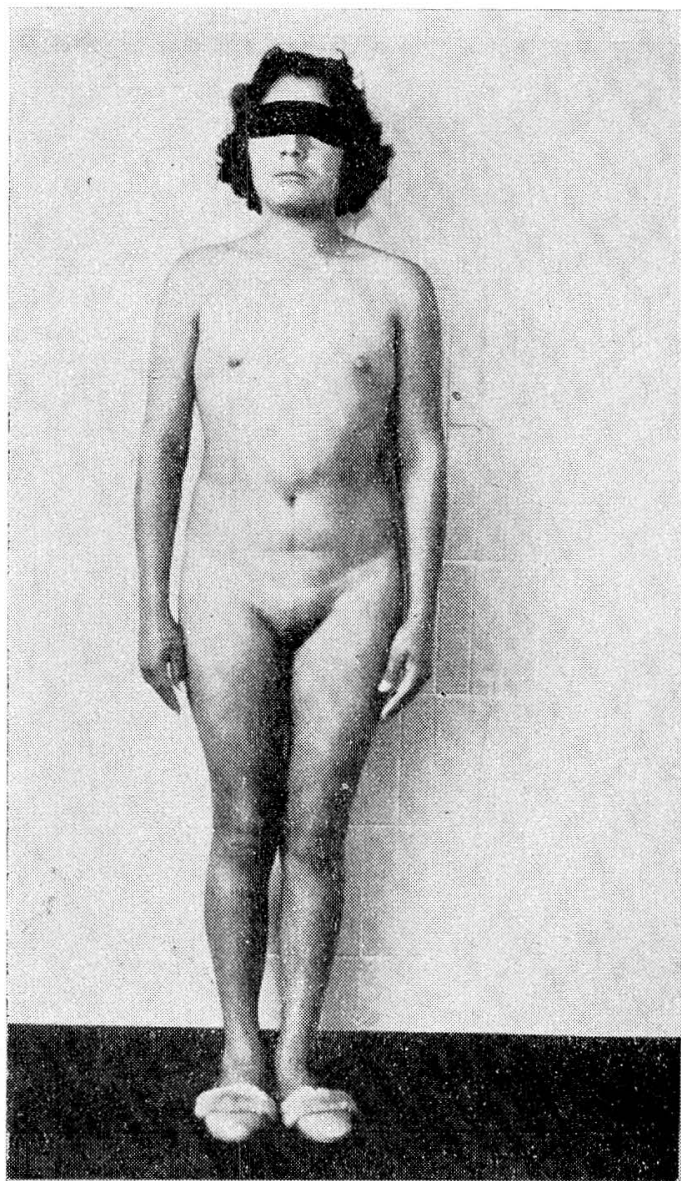

FIGURA 3 - Obsérvese el habitus masculino, sin desarrollo mamario y el crecimiento del elítoris. (Caso descrito previamente. Amer. J. Obstet. Gynec. 1971).

bién se debe hacer una clitorotomía y a continuación instituir tratamiento estrogénico.

3. Insensibilidad de los tejidos efectores. Existen 2 condiciones en las cuales se ha podido establecer una falla del tejido efector al efecto de una hormona.

1. Síndrome de testículo feminizante.
2. Insensibilidad ovárica al estímulo trópico.

1. El cuadro clínico característico del síndrome de testículo feminizante consiste en un individuo con buen desarrollo mamario, ausencia de veIlo sexual, infantilismo de genitales externos, vagina que termina en "fondo de saco", y en algunos casos, presencia de tumoración en los labios mayores o en los trayectos in- 


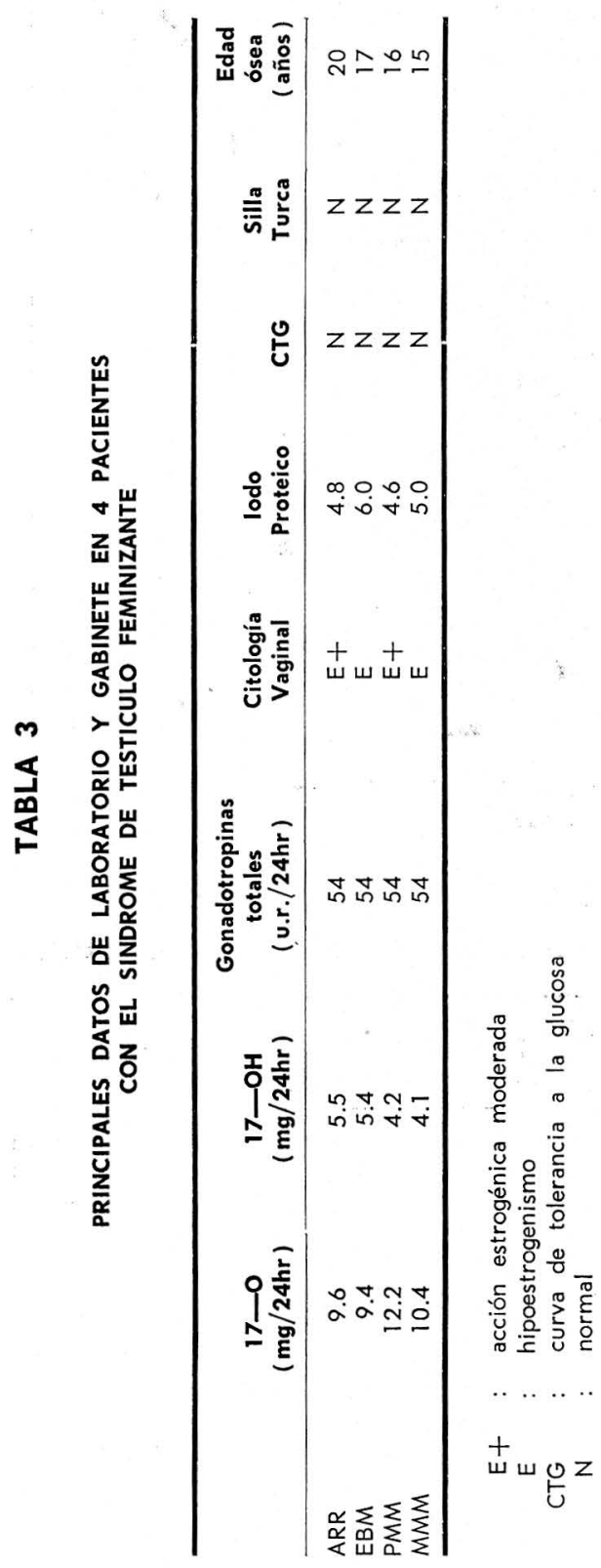

guilales, así como hernia de la misma región (Tabla 3 ). El motivo más frecuente de consulta es por amenorrea primaria; también por esterilidad y dispareunia. El análisis citogenético revela una cromatina sexual negativa y un cariotipo 46, XY (Fig. 4). En estos casos existen testículos que son capaces de producir la sustancia que inhibe a las estructuras de Müller; aunque también secretan andrógenos, aparentemente los tejidos del organismo son insensibles a su efecto. Se ha propuesto que el padecimiento resulta de un defecto enzimático que impide la conversión de testosterona a una forma activa, pero aún existen puntos oscuros a este respecto (9). El tratamiento consiste en la extirpación de los testículos y administración de estrógenos; en ocasiones es necesario realizar una plastia de la vagina. Este tipo de pacientes llega a tener una vida marital normal y la adopción es recomendable (Tabla 4 ).

2. Insensibilidad del aparato folicular al estímulo: es un padecimiento aún mucho más raro ya que sólo unos cuantos casos existen descritos en la literatura médica (10). Se trata de pacientes con amenorrea primaria e infantilismo sexual, con gonadotropinas hipofisiarias elevadas pero con ovarios de tipo prepuberal (Tabla 5). El diagnóstico se confirma cuando la administración de gonadotropinas exógenas tampoco es capaz de ejercer un efecto sobre los ovarios (Tabla 6). Aunque se han invocado varias teorías para explicar el trastorno, una de tipo inmunológico es la que ha recibido mayor atención. En la actualidad el único tratamiento disponible es la terapia de substitución con estrógenos adicionados con progesterona para inducir sangrados menstruales. La esterilidad es insoluble. 


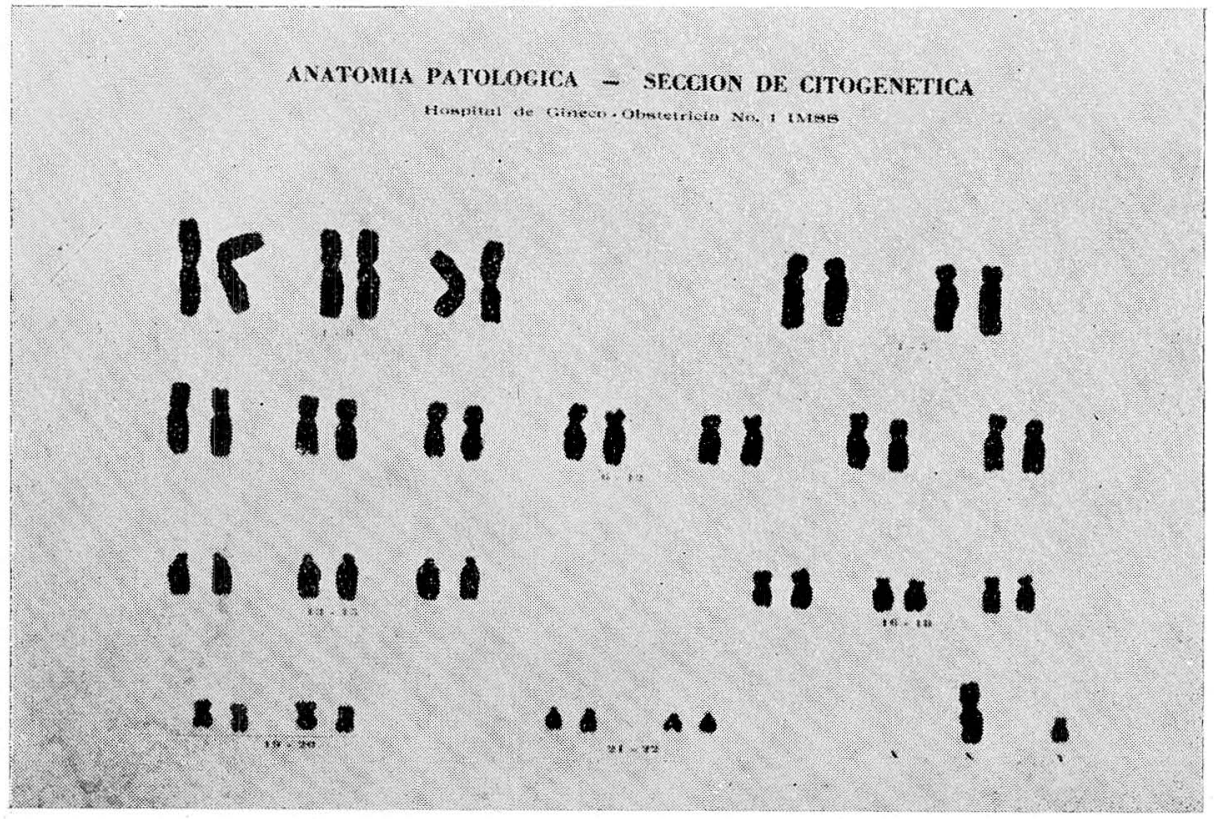

FIGURA 4 - Composición cromosómica 46, XY (normal del sexo masculino).

TABLA 4

ESTUDIO CITOGENETICO EN 4 PACIENTES CON EL SINDROME DE TESTICULO FEMINIZANTE

\begin{tabular}{lcccc}
\hline Paciente & $\begin{array}{c}\text { Cromatina Sexual } \\
\text { (mucosa bucal } \\
\text { leucocitos })\end{array}$ & $\begin{array}{c}\text { Cariotipo } \\
\text { (leucocitos) } \\
(\mathbf{5 0} \text { mitosis })\end{array}$ & Dedos & Dermatoglifos \\
\hline ARR & negativa & normal $46, X Y$ & normales & normales \\
EBM & negativa & normal $46, X Y$ & normales & normales \\
PMM & negativa & normal $46, X Y$ & normales & normales \\
MMM & negativa & normal $46, X Y$ & normales & normales \\
\hline
\end{tabular}

4. Amenorrea Primaria y Ovarios Poliquísticos. Algunas pacientes con o sin hirsutismo pueden no presentar su pubertad y el único hallazgo patológico que se encuentra es la presencia de ovarios bilateralmente crecidos (Fig. 5), con características histológicas semejantes a las descritas para el síndrome de Stein-Leventhal. Sin embargo, en los casos en que se ha llegado a practicar una resección cuneiforme de ovarios no se producen menstruaciones ni embarazos, lo cual indica que no se trata del mismo trastorno (Tabla 7). El estudio de laboratorio de estos casos no señala características especiales (Tabla 8), inclusive el estudio citogenético ha resultado de acuerdo con el sexo femenino. Con los cono- 
TABLA 5

PRINCIPALES CARACTERISTICAS CLINICAS EN PACIENTES

CON INSENSIBILIDAD AL ESTIMULO GONADOTROPICO

\begin{tabular}{|c|c|c|c|c|c|c|c|c|c|}
\hline Caso & Edad & $\begin{array}{l}\text { Menarquia } \\
\text { inducida } \\
\text { c/hormonas } \\
\text { sexuales } \\
\text { (edad) }\end{array}$ & $\begin{array}{c}\text { Menstruación } \\
\text { espontánea }\end{array}$ & Fenotipo & Talla $(\mathrm{m})$ & $\begin{array}{c}\text { Mamas } \\
\text { (desarrollo) }\end{array}$ & $\begin{array}{l}\text { Vello } \\
\text { sexual }\end{array}$ & $\begin{array}{l}\text { Genitales } \\
\text { externos }\end{array}$ & $\begin{array}{l}\text { Datos de mixedema } \\
\text { y/o deficiencia } \\
\text { suprarrenal }\end{array}$ \\
\hline 1 & 26 & 17 & nunca & femenino & 1.60 & escaso & normal & normales & no \\
\hline 2 & 26 & 16 & nunca & femenino & 1.50 & escaso & normal & normales & no \\
\hline
\end{tabular}

TABLA 6

PRUEBA DE ESTIMULACION EN UNA PACIENTE

CON INSENSIBILIDAD OVARICA AL ESTIMULO GONADOTROPICO

\begin{tabular}{|c|c|c|c|c|c|c|c|c|}
\hline & $17-0$ & $\begin{array}{l}\text { EXCRECION } \\
\text { Pdiol } \\
\mathrm{mg} / \mathbf{2 4} \mathrm{hr}\end{array}$ & $\begin{array}{l}\text { URINARIA } \\
\text { Ptriol }\end{array}$ & $\begin{array}{c}E T \\
(u g / 24 h r)\end{array}$ & $\begin{array}{l}\text { MOCO } \\
\text { filantez } \\
(\mathrm{cm})\end{array}$ & $\begin{array}{l}\text { CERVICAL } \\
\text { arborización } \\
(+\mathbf{a}+++)\end{array}$ & Endometrio & $\begin{array}{l}\text { Citología Vaginal } \\
\text { (Indice de } \\
\text { maduración ) }\end{array}$ \\
\hline $\begin{array}{l}\text { Basal } \\
5^{\circ} \text { día Pergonal-500 }\end{array}$ & 10.0 & 0.4 & 0.2 & 0.0 & 2 & + & inactivo & $10 / 80 / 10$ \\
\hline (150 UI-FSH/150 UI-LH/día) & 7.0 & 0.4 & 0.3 & 0.0 & 3 & + & Inactivo & $15 / 85 / 0$ \\
\hline
\end{tabular}




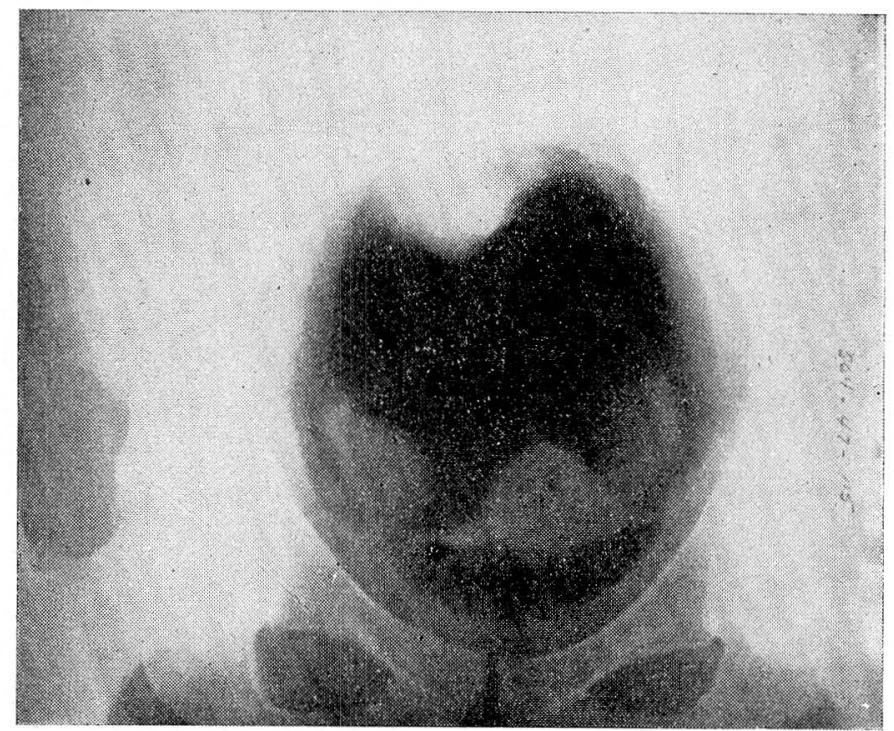

FIGURA 5 - Radiológicamente se demuestra con crecimiento ovárico bilateral.

TABLA 7

CARACTERISTICAS CLINICAS EN 4 PACIENTES CON AMENORREA PRIMARIA $Y$ OVARIOS POLIQUISTICOS

\begin{tabular}{lccccccc}
\hline Paciente & $\begin{array}{c}\text { Edad } \\
\text { (años) }\end{array}$ & Hirsutismo & Obesidad & $\begin{array}{c}\text { Glándulas } \\
\text { mamarias }\end{array}$ & Utero & $\begin{array}{c}\text { Genitales } \\
\text { externos }\end{array}$ & S.U.D.H. \\
\hline LRL & 18 & sí & no & $\mathrm{N}$ & $\mathrm{D}$ & $\mathrm{N}$ & sí \\
CMV & 23 & no & no & $\mathrm{D}$ & $\mathrm{N}$ & $\mathrm{N}$ & sí \\
RMR & 24 & no & no & $\mathrm{D}$ & $\mathrm{D}$ & $\mathrm{N}$ & sí \\
EDR & 36 & no & no & $\mathrm{D}$ & $\mathrm{N}$ & $\mathrm{N}$ & sí \\
(N) Normal & (D) Disminuídas de tamaño & & & \\
(S.U.D.H.) & Sangrado uterino por deprivación hormonal & & & \\
\hline
\end{tabular}

cimientos actuales no se ha podido establecer la fisiopatogenia del trastorno.

\section{Pubertad Precoz.}

Es difícil definir la pubertad pre$\mathrm{coz}$ por la gran variación que existe en cuanto a la edad de aparición de la pubertad. Se puede considerar, arbitrariamente, como la aparición de un desarrollo mamario y vello se- xual antes de los 8 años de edad, o como la ocurrencia de menarquia antes de los 9 años; habitualmente produce cambios muy significativos en el desarrollo psicológico y físico del niño. El desarrollo precoz de las características sexuales secundarias y la activación prematura del ciclo menstrual pueden ocasionar trastornos en el desarrollo de la personalidad. 


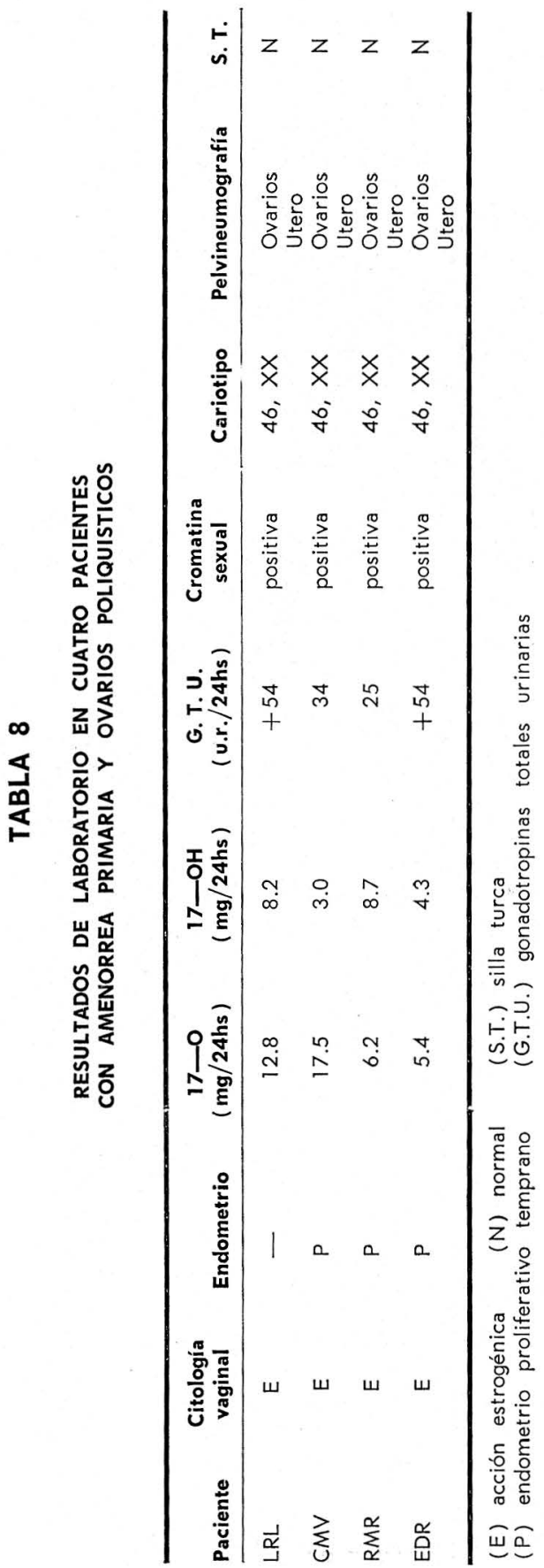

Generalmente se aceptan dos tipos de pubertad precoz: la verdadera y la falsa. En la precoz "verdadera" se presentan, en la misma forma y secuencia, los cambios que ocurren en la pubertad normal; este trastorno es más frecuente en las niñas y puede obedecer a muchas causas. En la "falsa" pubertad precoz, se presenta una maduración de los genitales externos y un desarrollo prematuro de los caracteres sexuales secundarios; sin embargo, el ovario no alcanza su madurez y por consiguiente no hay ovulación. Esta variedad de pubertad precoz puede ser de dos tipos: isosexual y heterosexual, de acuerdo con las características sexuales que se encuentran y por lo tanto con el tipo de hormona circulante.

Una clasificación de pubertad precoz puede ser la siguiente:

\section{I - Verdadera.}

A. Constitucional.

B. Asociada a otra patología.

1. Displasia Fibrosa Poliostótica.

2. Hipotiroidismo juvenil.

C. Lesión del sistema nervioso central.

a) Postencefalitis o postmeningitis.

b) hidrocéfalo

c) lesión traumática

d) tumoral (pinealoma, hamartona, granulosa)

e) encefalopatía degenerativa

f) esclerosis tuberosa

g) enfermedad de Von Recklinghausen

h) aracnoiditis quística. 


\section{II - Falsa.}

A. Por aumento de hormonas sexuales.

1. Isosexual.

a) Neoplasia ovárica (teca-granulosa, teratoma, coriocarcinoma).

b) Ingestión de estrógenos.

2. Heterosexual.

a) Neoplasia suprarrenal.

b) Hiperplasia suprarrenal congénita.

c) Ingestión de andrógenos.

B. Aumento de la sensibilidad del órgano efector.

1. Telarquia precoz.

2. Pubarquia precoz.

Pubertad precoz constitucional. Es más frecuente en las niñas y en ocasiones se ha encontrado un carácter familiar. El diagnóstico de este trastorno se hace siempre después de haber excluído patología orgánica, aunque no es infrecuente que se encuentren los ovarios de tipo poliquístico. Aunque el desarrollo sexual se ha adelantado, en cambio el desarroIlo dental, psicológico e intelectual están de acuerdo con la edad cronológica. El desarrollo óseo, bajo la influencia hormonal, se adelanta, y las personas terminan siendo de talla baja. La fertilidad y la menopausia son semejantes a las de las mujeres normales (12).

Displasia Fibrosa Poliostótica. Este padecimiento se caracteriza por lesiones óseas diseminadas y placas cutáneas de color café el cual puede acompañarse en el 40 por ciento de los casos con una pubertad precoz. La explicación para tal asociación se desconoce y todos los exámenes hor- monales que se han realizado han resultado normales (13).

Mixedema. Aunque en la mayor parte de los casos de mixedema existe un retardo en el desarrollo sexual, en ocasiones el hipotiroidismo primario se asocia con pubertad precoz, galactorrea e hiperpigmentación. Se ha propuesto que en tales casos la falla tiroidea ocasionaría un aumento en la secreción de TSH y esto a su vez una hipersecreción total adenohipofisiaria (14).

Lesión en el sistema nervioso central. Aunque la patología responsable es muy variada, el común denominador es una secreción de gonadotropinas hipofisiarias que estimula al ovario. Para hacer el diagnóstico es necesario recurrir a todo el arsenal de pruebas radiológicas y neurológicas. Merece un comentario especial, por el interés que ha despertado, la asociación de tumores de la pineal con pubertad precoz. En una revisión de la literatura se encontró que en realidad esos tumores no eran de origen pineal sino teratomas 0 se originaban de las estructuras de sostén de la pineal. Posteriormente se demostró que la pineal produce melatonina que es una hormona derivada de la serotonina.

El mecanismo patogénico por el cual se produce pubertad precoz con tumores de la pineal 0 de sus estructuras de sostén, no se ha aclarado. El efecto pudiera no estar relacionado con la actividad hormonal de la glándula, sino ser consecuencia de un efecto físico del tumor sobre el hipotálamo posterior. Todavía no se ha podido explicar por qué los tumores de la pineal que se asocian con pubertad precoz sólo han ocurrido en el sexo masculino (15).

Pseudopubertad por aumento de las hormonas sexuales. Los tumores 
ováricos son la primera causa a considerar en el diagnóstico diferencial de pseudopubertad isosexual. Estos tumores son raros y aún más el hecho de que poseen actividad endocrina. Los tumores de la teca-granulosa habitualmente son de gran tamaño, $y$ por lo tanto, muy aparentes en el abdomen y a la exploración rectal; producen grandes cantidades de estrógenos que frecuentemente originan cuadros de sangrado vaginal muy importante. Las gonadotropinas hipofisiarias en estos casos se encuentran ausentes. Los tumores son habitualmente benignos; en cambio, cuando se trata de un coriocarcinoma, éste tiene gran malignidad y la determinación de hormona coriónica ayuda en el diagnóstico diferencial.

En los casos de pseudopubertad heterosexual, los tumores suprarrenales y la hiperplasia suprarrenal se caracterizan por un cuadro clínico de pseudohermafroditismo en el que es posible demostrar una excreción elevada de 17-cetoesteroides y pregnandiol. Finalmente, la ingestión de drogas con actividad hormonal se debe tener siempre en cuenta para el diagnóstico diferencial; los niños pueden ingerir accidentalmente estrógenos prescritos a los adultos; también existen algunos medicamentos que se han contaminado con estrógenos y así se han informado casos de pubertad precoz después de la ingestión de la hidrazida del ácido isonicotínico y de vitaminas. La pseudopubertad heterosexual también se ha observado frecuentemente con el uso de drogas anabólicas esteroideas.

Hipersensibilidad del órgano efector. La telarquia precoz consiste en cierto desarrollo mamario no acompañado de otros signos de maduración sexual; tampoco se encuentran gonadotropinas ni estrógenos circulantes; la telarquia persiste hasta la época de la pubertad y no modifica el tiempo de aparición de ésta.

El desarrollo prematuro del vello sexual se conoce como pubarquia precoz y no se acompaña de otros signos de madurez sexual. Tampoco existe hirsutismo ni clitoromegalia. Los exámenes hormonales de laboratorio no muestran anormalidades, ni la edad ósea se encuentra acelerada.

Tratamiento de al pubertad precoz. El tratamiento de la pubertad precoz depende de la naturaleza del trastorno y de la edad en que consulta la niña. En la telarquia precoz no se debe instituir tratamiento médico ni hacer estudios extensos puesto que la condición es benigna. Lo mismo se aplica a la pubarquia precoz. Tratamiento etiológico con cortisona y con tiroides debe administrarse en casos de hiperplasia suprarenal congénita y mixedema respectivamente. Los tumores ováricos, suprarrenales e hipofisiarios y del sistema nervioso deben extirparse según sea el caso. La pubertad precoz de tipo constitucional se ha tratado en los últimos años, basándose en el principio de que una substancia que inhiba a las gonadotropinas hipofisiarias, indirectamente hace desaparecer las manifestaciones de actividad ovárica secundaria. Así se ha usado extensamente, aunque con resultados variables, un progestágeno potente, el acetato de medroxiprogesterona.

También merece comentario especial un aspecto que no debe descuidarse: el manejo psicológico de las pacientes con pubertad precoz, para evitar así los trastornos de personalidad que habitualmente se instalan en estas niñas. 


\section{III - Síndrome Virilizante.}

Estrictamente no se trata de un problema de la adolescencia ya que habitualmente son casos en los que el virilismo está presente desde el nacimiento, pero por no habérsele prestado atención o por haber ido progresando en severidad, es hasta la adolescencia cuando al no aparecer la menarquia se lleva a cabo la consulta médica. En tales casos se puede tratar de una disgenesia gonadal mixta, un hermafroditismo, o una hiperplasia suprarrenal congénita moderada. Por otro lado, el virilismo súbito en la adolescencia puede originarse por un tumor suprarrenal $u$ ovárico. La intensidad de la virilización puede ser muy variable: 1) hirsutismo y clitoromegalia, como ocurre en los tumores y en la hiperplasia suprarrenal y 2) clítoromegalia aislada, como en la disgenesia gonadal mixta, en tumores de gónada disgenética $y$ en el hermafroditismo verdadero. En todos estos casos la fisiopatogenia se explica por la presencia de andrógenos circulantes en cualquier época de la vida intrauterina o después del nacimiento. El tratamiento consiste en la extirpación del clítoris y el acondicionamiento para una vida social y sexual de acuerdo con el sexo femenino.

\section{IV - Amenorrea primaria y desarrollo sexual normal.}

En ocasiones, raras por cierto, la mujer presenta un desarrollo de sus caracteres sexuales secundarios pero la menarquia no hace su aparición. En tales circunstancias se debe sospechar una falla del órgano efector: el útero; el diagnóstico se confirma cuando, con himen perforado, la administración de un ciclo con estrógenos y progesterona no produce un sangrado por deprivación hormonal.

TABLA 9

PRINCIPALES CARACTERISTICAS CLINICAS EN PACIENTES CON ANCM:ALIAS MULLERIANAS

\begin{tabular}{rccccccc}
\hline $\begin{array}{c}\text { Caso } \\
\text { No. }\end{array}$ & Edad & $\begin{array}{c}\text { Talla } \\
(\mathbf{m})\end{array}$ & $\begin{array}{c}\text { Peso } \\
(\mathbf{K g})\end{array}$ & $\begin{array}{c}\text { Desarrollo } \\
\text { Mamario }\end{array}$ & $\begin{array}{c}\text { Vello } \\
\text { Sexual }\end{array}$ & $\begin{array}{c}\text { Exploración } \\
\text { Vagina }\end{array}$ & $\begin{array}{c}\text { Ginecológica } \\
\text { Utero }\end{array}$ \\
\hline 1 & 27 & 1.46 & 60 & Normal & Normal & $1 / 3$ inferior & Ausente \\
2 & 16 & 1.52 & 43 & Normal & Normal & $1 / 5$ inferior & Ausente \\
3 & 23 & 1.47 & 58 & Normal & Normal & $1 / 5$ inferior & Ausente \\
4 & 24 & 1.59 & 71 & Normal & Normal & $1 / 3$ inferior & Ausente \\
5 & 23 & 1.52 & 42 & Normal & Normal & $1 / 3$ Inferior & Dudoso \\
6 & 26 & 1.56 & 60 & Normal & Normal & $1 / 5$ inferior & Ausente \\
7 & 26 & 1.45 & 57 & Normal & Normal & $1 / 5$ inferior & Ausente \\
8 & 21 & 1.52 & 48 & Normal & Normal & $1 / 3$ inferior & Ausente \\
9 & 29 & 1.58 & 64 & Normal & Normal & $1 / 3$ inferior & Ausente \\
10 & 16 & 1.58 & 63 & Normal & Normal & $1 / 3$ inferior & Ausente \\
11 & 33 & 1.54 & 59 & Normal & Normal & $1 / 3$ inferior & Ausente \\
\hline
\end{tabular}

Dos son los padecimientos con tales características:

1. Ausencia congénita del útero (anomalía Mülleriana).

2. Destrucción del endometrio.

\section{Anomalías Müllerianas.}

Se trata de un defecto congénito, de naturaleza desconocida y aparentemente no relacionado con trastornos cromosómicos (Tabla 9). La 
Vol. XXII

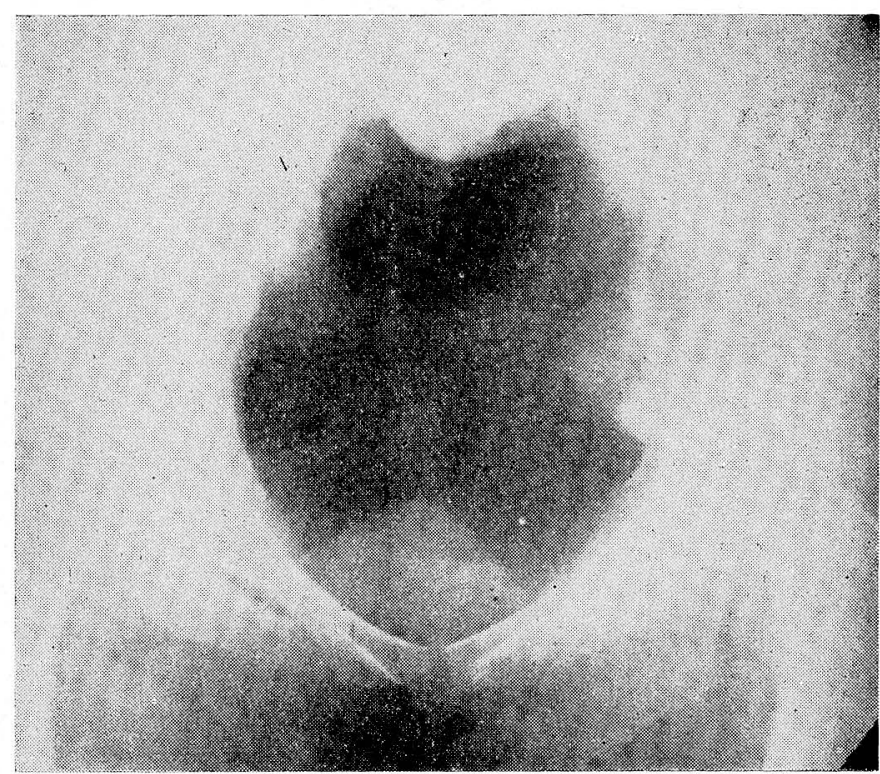

FIGURA 6 - No se observa la sombra uterina. Nótese la existencia de gónadas a los lados de la pelvis.

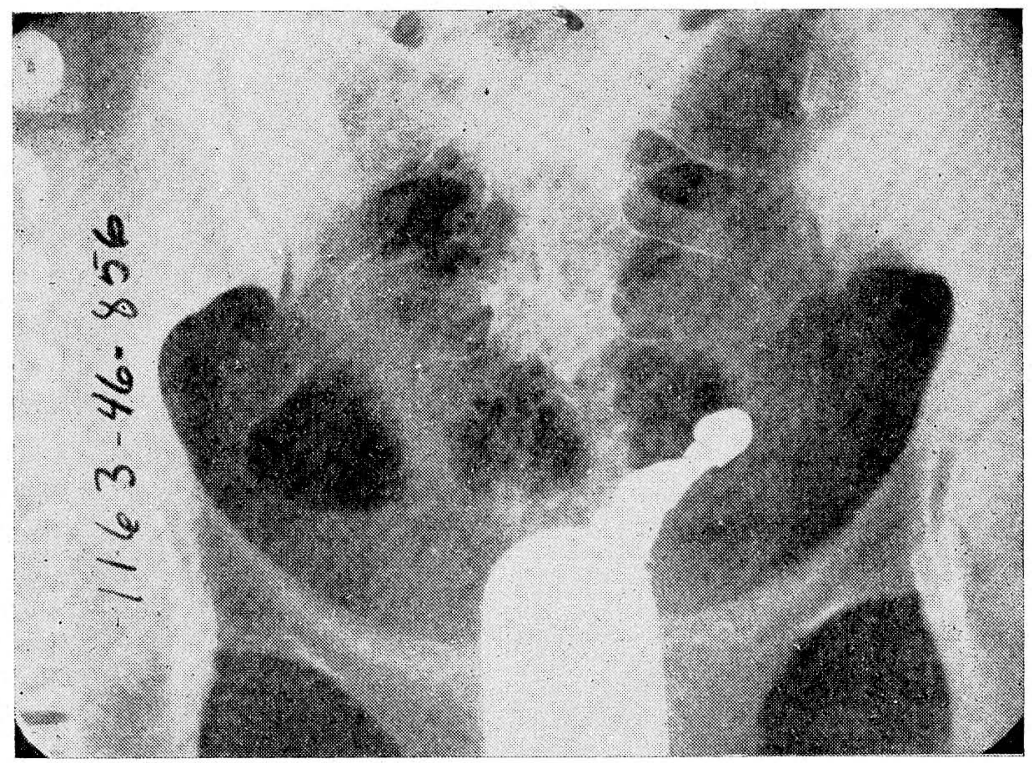

FIGURA 7 - Imagen radiológica típica de tuberculosis endometrial. 


\section{TABLA 10}

PRINCIPALES DATOS DE LABORATORIO, GABINETE Y HALLAZGOS QUIRURGICOS

\begin{tabular}{|c|c|c|c|c|c|c|c|}
\hline Caso $\mathrm{N}^{0}$ & Ovulación & $\begin{array}{l}\text { ESTUDIOS } \\
\text { Pelvineumografía }\end{array}$ & $\begin{array}{c}\text { RADIOLOGICOS } \\
\text { Urografía } \\
\text { Descendente }\end{array}$ & $\begin{array}{l}\text { Columna } \\
\text { Vertebral }\end{array}$ & $\begin{array}{c}\text { Cromatina } \\
\text { Sexual }\end{array}$ & Cariotipo & $\begin{array}{l}\text { Hallazgos } \\
\text { Quirúrgicos }\end{array}$ \\
\hline 1 & $\begin{array}{l}\text { Pregnandiol } \\
\text { y CTB }\end{array}$ & $\begin{array}{l}\text { Ausencia de útero } \\
\text { Ovarios normales }\end{array}$ & Normal & Normal & - & 46. $X X$ & $\begin{array}{l}\text { Agenesia Uterina } \\
\text { Ovarios Normales }\end{array}$ \\
\hline 2 & $\begin{array}{l}\text { Cuerpo Lúteo } \\
\text { (biopsia) }\end{array}$ & — & Normal & Normal & $33 \%$ & $46, X X$ & $\begin{array}{l}\text { Agenesia Uterina } \\
\text { Ovarios Normales }\end{array}$ \\
\hline 3 & - & $\begin{array}{l}\text { Ausencia de útero } \\
\text { Ovarios normales }\end{array}$ & Normal कि & Normal & $33 \%$ & 46, $x x$ & $\begin{array}{l}\text { Agenesia Uterina } \\
\text { Ovarios Normales }\end{array}$ \\
\hline 4 & - & $\begin{array}{l}\text { Ausencia de útero } \\
\text { Ovarios normales }\end{array}$ & Normal & - & $27 \%$ & $46, x \times$ & — \\
\hline 5 & $\begin{array}{l}\text { Cuerpo Lúteo } \\
\text { (biopsia) }\end{array}$ & - & Normal & - & $46 \%$ & $46, x \times$ & $\begin{array}{l}\text { Agenesia Uterina } \\
\text { Ovarios Normales }\end{array}$ \\
\hline 6 & - & $\begin{array}{l}\text { Ausencia de útero } \\
\text { Ovarios normales }\end{array}$ & Normal & Normal & $30 \%$ & $46, x X$ & - \\
\hline 7 & - & $\begin{array}{l}\text { Ausencia de útero } \\
\text { Ovarios normales }\end{array}$ & $\begin{array}{l}\text { Ausencia riñón } \\
\text { izquierdo }\end{array}$ & Normal & $12 \%$ & $46, x X$ & $\longrightarrow$ \\
\hline 8 & - & $\begin{array}{l}\text { Ausencia de útero } \\
\text { Ovarios normales }\end{array}$ & Normal & Normal & $22 \%$ & $46, x \times$ & - \\
\hline 9 & $\begin{array}{l}\text { Pregnandiol } \\
\text { y CTB }\end{array}$ & $\begin{array}{l}\text { Ausencia de útero } \\
\text { Ovarios pequeños }\end{array}$ & $\begin{array}{l}\text { Riñón pélvico } \\
\text { derecho }\end{array}$ & Espina bífida & $36 \%$ & 46, $x \times$ & - \\
\hline 10 & $\begin{array}{l}\text { Cuerpo Lúteo } \\
\text { (biopsia) }\end{array}$ & $\begin{array}{l}\text { Ausencia de útero } \\
\text { Ovarios normales }\end{array}$ & Normal & Normal & $30 \%$ & $46, x x$ & $\begin{array}{l}\text { Agenesia Uterina } \\
\text { Ovarios Normales }\end{array}$ \\
\hline 11 & $\begin{array}{l}\text { Pregnandiol } \\
\text { y CTB }\end{array}$ & $\begin{array}{l}\text { Ausencia de útero } \\
\text { Ovarios normales }\end{array}$ & Normal & Normal & $20 \%$ & $46, X X X$ & 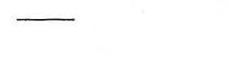 \\
\hline
\end{tabular}


anomalía puede afectar también a los oviductos y al tercio superior de la vagina. El diagnóstico se realiza fácilmente mediante una pelvineumografía (Fig. 6). En estos casos los ovarios funcionan normalmente y se pueden demostrar inclusive la presencia de ovulación (16) (Tabla 10). Cuando involucra a la vagina es motivo frecuente de dispareunia y entonces se hace necesario una plastia quirúrgica de la misma.

\section{Lesión Endometrial.}

La tuberculosis genital que habitualmente se inicia en trompas puede alcanzar el endometrio y destruirlo desde su capa basal (Fig. 7). El pronóstico en cuanto a la recuperación después de tratamiento antifímico es muy malo y ésto hace que la amenorrea persista y la fertilidad sea nula (17). El diagnóstico se puede hacer indirectamente con una histerosalpingografía y cultivo de secreción endometrial.

\section{Comentario Final}

Los trastornos ginecoendocrinos de la adolescencia reclaman toda la atención con el objeto de reconocer lo más tempranamente posible su naturaleza y así proporcionar el tratamiento adecuado para lograr una feminización correcta y una vida sexual normal en el futuro.

\section{Final Remarks}

The gyneco-endocrino disorders during adolescence claim full attention for the purpose of determining their nature as early as possible and thus be able to apply adequate treatment to attain correct feminization and a normal future sexual life.

\section{BIBLIOGRAFIA}

1 ZARATE A., ESTEVEZ R., ORTIZ J., CASTELAZO-AYALA L.: Ginec. Obstet. Mex. 28: $111,1970$.

2 ODELL WO, MOYER DL, Physiology of Reproduction. C. V. Mosby Company, Saint Louis, 1971.

3 GUYDA HJ, JOHANSON AJ, MIGEON CJ, BLIZZARD RM. Pediat. Res. 3: 533, 1969.

4 ZARATE A, CANALES ES, RUIZ F, CASTELAZO-AYALA L. Inter. J. Gynaec. Obstet. 9: $155,1971$.

5 CABALLERO RE, RUIZ F, ZARATE A, LOPEZ-AMOR E. SOC. Mex. Nut. Endocr. 10: 291,1970

6 ZARATE A, KASTIN AJ, SORIA J, SCHALLY $A V$, En Prensa.

7 ZARATE A, ESTEVEZ R, GARCIA REYES JA, CASTELAZO-AYALA L. Rev. Invest. Clin. 20 : $101,1968$.

8 ZARATE A, KARCHMER S, ESTEVEZ R, TETER J. Amer. J. Obstet. Gynaec. 110: 875, 1971.

9 ZARATE A, KARCHMER S, GOMEZ E, ESTEVEZ R, GARCIA REYES JA. Rev. Invest. Clin. 22: 53, 1970.

10 ZARATE A, VALENZUELA $S$, CANALES ES. Ginec. Obstet. Mex. 28: 409, 1970.

11 CANALES ES, ZARATE A, CASTELAZO-AYALA L. Obstet. Gynec. 307: 205, 1971.

12 COHEN RA, WURTMAN RJ, AXELROD J. and Snyder SH. Ann. Inter. Med. 61: $1444,1964$.

13 ALBRIGHT F, REIFENSTEIN EC: The Parar thyroid glands and Metabolic Bone Disease. Selected studies. Williams Wilkins Co. Baltimore 1948.

14 VAN WYK JJ, GRUMBACH M. J. Pediat. 57: $416,1960$.

15 LIST CF, DOWMAN CE, BAGCHI BK, DEBIN J. Neurology. 8: 164, 1964.

16 RICAUD L., CANALES ES, ALAYA L, ZARATE A. Ginec. Obstet. Mex. 29: 281, 1971.

17 SALAS D, GARCILAZO J, ZARATE A. Ginec. Obstet. Mex. 26: 67, 1969. 